\title{
Complex Formation of Poly-L-lysine with Sodium Cellulose Sulfate
}

\author{
Katsuro Shinoda, Toshio Hayashi, and Akio Nakajima* \\ Department of Polymer Chemistry, Kyoto University, \\ Sakyo-ku, Kyoto 606, Japan. \\ (Received October 28, 1975)
}

\begin{abstract}
The interactions between poly-L-lysine (PLL) and sodium cellulose sulfate (SCS) have been studied as functions of both the $\mathrm{pH}$ and the PLL-to-SCS ratio by using circular dichroism spectroscopy. SCS reacted with PLL and induced the formation of the $\alpha$-helix of PLL. The induced helical content was proportional to the SCS mole fraction in the complex, but deviated from the stoichiometrical relationship. In this system, no disturbing effects on the formation of $\alpha$-helix of PLL were observed. These results were different from those for the mucopolysaccharides-PLL systems reported by Gelman, et al., in some respects, and could be interpreted by taking into account the chain conformations and the location of sulfonic groups along the SCS backbone chains.
\end{abstract}

KEY WORDS Polyelectrolyte Complex / Poly-L-Lysine / Sodium Cellulose Sulfate / Conformations / Circular Dichroism /

In our previous reports, ${ }^{1-3}$ we have investigated the effect of the conformations and the configurations of the polyacidic component on the interactions with poly-L-lysine (PLL). In this paper, to extend our study to more complex systems, an acidic polysaccharide, sodium cellulose sulfate (SCS), was used as an acidic component. Gelman, et al., ${ }^{4-8}$ reported the interactions between various mucopolysaccharides and cationic polypeptides in aqueous solution at neutral $\mathrm{pH}$ by using the circular dichroism technique, as models for connective tissue systems. They found that both the stoichiometry and the strength of the interactions depended on the position, number, and type of anionic groups attached to the polysaccharide backbone. In addition, maximum interaction occurred at a definite residue ratio of amino acid to disaccharide, which was characteristic for each mucopolysaccharide.

However, our results for the SCS-PLL system showed some differences from their results. We will discuss these differences by comparison with our previous papers.

* To whom correspondence should be addressed.

\section{EXPERIMENTAL}

\section{Materials}

Poly-L-lysine and acridine orange (AO) were the same samples as those previously described. ${ }^{1,2}$ Sodium cellulose sulfate (Lot, KELCO-LV-1) was obtained from Kelco Co., Ltd., and was dialysed after precipitations with ethanol before use. The molecular weight of SCS was 67,000 from the limiting viscosity number $[\eta]$ in $2-N$ $\mathrm{NaOH}$ at $25^{\circ} \mathrm{C}$. The content of ionizable groups, $\mathrm{HSO}_{3}$, in SCS was 2.5 per pyranose residue.

\section{Preparation of Mixtures}

Separate dilute aqueous solutions were prepared for PLL and SCS. The PLL solution was adjusted at $\mathrm{pH} 12$ by adding $1.0-\mathrm{N} \mathrm{NaOH}$. Mixtures were prepared by slow dropwise addition of an aqueous solution of SCS at $\mathrm{pH} c a .7$ to the

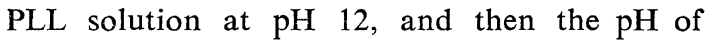
the mixtures were brought to the desired values by adding $\mathrm{HCl}$. The relative proportions of the two polymer components are represented by the mole ratio of the ionizable groups, i.e., the concentration of amino acid residue relative to the molar concentration of the sulfonic group of SCS. Most of the mixtures gave a clear solution, but a few mixture solutions showed 
slight turbidity. A $53: 47$ mole ratio PLL-SCS mixture at $c a$. $\mathrm{pH} 8$ gave a clear solution for several days after preparation, but became weakly turbid with the lapse of time. However, the circular dichroism spectra were only slightly influenced by the turbidity. A weakly turbid final solution stored $c a$. 30 days at room temperature showed a $c a .5 \%$ decrease in the numerical value of residue ellipticity compared with the initial clear solution. This result shows that the CD spectra would be only slightly affected by the turbidity.

\section{Circular Dichroism}

The circular dichroism spectra were measured at $25 \pm 0.5^{\circ} \mathrm{C}$ using a JASCO J-20 CD/ORD Spectropolarimeter equipped with a quartz cell of path length $1 \mathrm{~mm}$. The PLL concentrations in the mixtures were from 0.002 to $0.003 \mathrm{M}$. The concentrations were determined by conductometric titration and/or gravimetry. The residue ellipticity $[\theta]$, in degree $\cdot \mathrm{cm}^{2} \cdot \mathrm{dmol}^{-1}$, for the mixtures was calculated based on the mole concentration of the PLL.

\section{pH Measurements}

The $\mathrm{pH}$ values of the solutions were measured with a Hitachi-Horiba pH-Meter Model F-7 equipped with a combination $\mathrm{pH}$ electrode 6028$10 \mathrm{~T}$, the sensitivity of which was $0.005 \mathrm{pH}$.

\section{RESULTS AND DISCUSSION}

\section{$C D$ Spectra of Mixtures}

The addition of SCS to PLL at acidic or neutral $\mathrm{pH}$ caused a change in the "charged coil" conformation of PLL, which is reflected in the $C D$ spectra. Figure 1 shows the $C D$ spectra expressed in the residue ellipticity $[\theta]$ of PLL_SCS mixtures obtained from typical experiments at low $\mathrm{pH}$ region, where PLL takes the charged coil conformation in the absence of SCS. Figure 2 shows the CD spectra of the 77 : 23 mole ratio PLL-SCS mixtures at various pH's. SCS as well as other simple polysaccharides shows no characteristic $C D$ spectrum in the region of wavelength above $200 \mathrm{~nm}$. Therefore, these spectra show the conformation of PLL in the mixture. It can be seen that these curves in Figures 1 and 2 almost accord with those observed for PLL in aqueous solution by itself

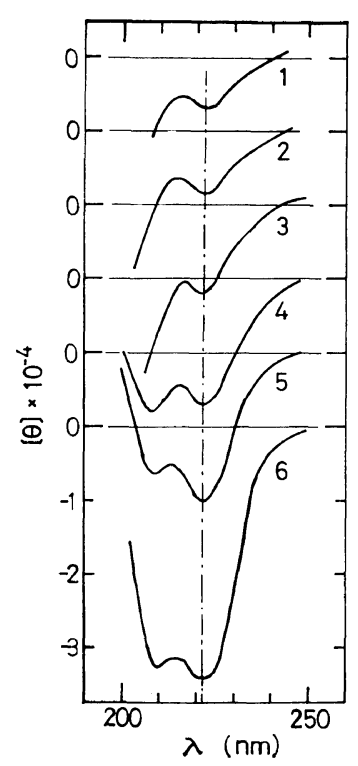

Figure 1. CD spectra of PLL-SCS mixtures at low $\mathrm{pH}$ values and at $25^{\circ} \mathrm{C}$. The mole ratios of PLL to SCS and the pH's are: (1) $75: 25, \mathrm{pH}=$ 2.81; (2) $70: 30, \mathrm{pH}=2.41$; (3) $67: 33, \mathrm{pH}=2.08$; (4) $53: 47, \mathrm{pH}=3.12$; (5) $51: 49, \mathrm{pH}=2.48$; (6) 17 : $83, \mathrm{pH}=3.27$.

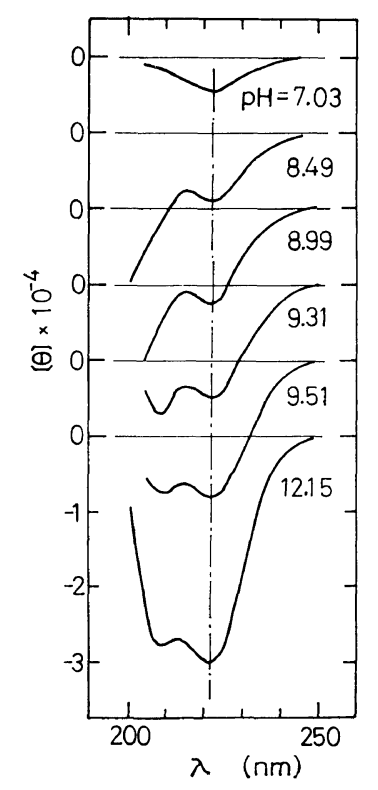

Figure 2. CD spectra of $77: 23$ mole ratio PLLSCS mixtures at $25^{\circ} \mathrm{C}$ and various $\mathrm{pH}$ values. 


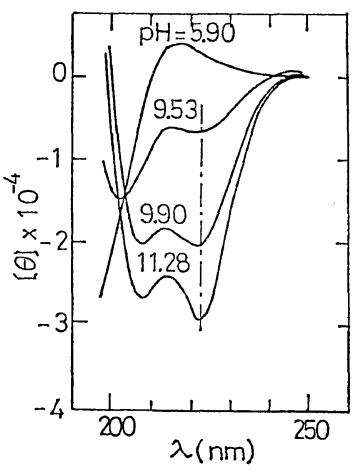

Figure 3. CD spectra of PLL in aqueous solution at $25^{\circ} \mathrm{C}$ and various $\mathrm{pH}$ values.

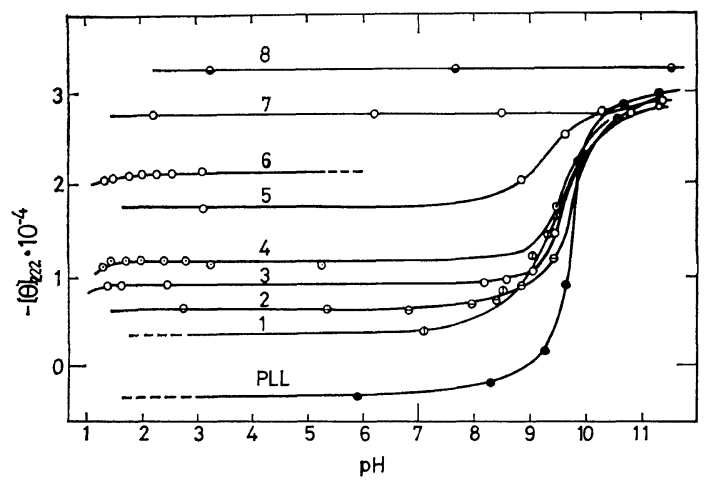

Figure 4. $-[\theta]_{222}$ plotted against $\mathrm{pH}$ for PLLSCS mixtures and for PLL at $25^{\circ} \mathrm{C}$. The mole ratios of PLL to SCS are: (1) $77: 23$; (2) $75: 25$; (3) $70: 30$; (4) $67: 33$; (5) $53: 47$; (6) $51: 49$; (7) 41 : 59; (8) $17: 83$.

(see Figure 3). The curves 5 and 6 in Figure 1, and the curves of $\mathrm{pH} 9.51$ and 12.15 in Figure 2 are typical spectra of PLL in $\alpha$-helix conformation. Other curves, all having the $222 \mathrm{~nm}$ peak, are quite similar in shape to those in the transition region of PLL, at which some proportion of the PLL is in the $\alpha$-helical conformation and the rest is in the "charged coil", form. This result indicated that the PLL in the complex takes the $\alpha$-helical or charged coil conformations in a similar manner to PLL by itself in aqueous solution. Thus, we could regard the numerical value of residue ellipticity at $222 \mathrm{~nm},[\theta]_{222}$, as an index of the helical content of the PLL in the complex.

pH Dependence of $-[\theta]_{222}$ of PLL-SCS Mixtures

In Figure $4,-[\theta]_{222}$ of mixtures containing different ratios of PLL and SCS is plotted against $\mathrm{pH}$; here data not shown in previous figures are also included. As is also illustrated in Figure 4, PLL shows a sharp transition at about $\mathrm{pH}$ 9.5, while the $\alpha$-helix region is broadened by the coexisting SCS. These induced $\alpha$-helices of PLL would be caused by the neutralization of the $\varepsilon$-amino groups by the sulfonic groups attached to SCS. The sulfonic group, a strong electrolyte, is fully ionized in the $\mathrm{pH}$ region studied here. Naturally, a transition region at around $\mathrm{pH} 9.5$ caused by the dissociation of the $\varepsilon$-amino groups is observed in the region below 0.5 of SCS mole fraction.

It is found that the numerical value of $-[\theta]_{222}$ of the mixures including a large excess of SCS was larger than that of PLL in $\alpha$-helical form. A similar phenomenon was observed in poly (acrylic acid) (PAA)-PLL system. ${ }^{2}$ This behavior was interpreted by considering that the nonhelical part existing in PLL even in the helical region may take a $\alpha$-helix conformation by the coexistence of the oppositely charged polyelectrolyte. A similar explanation may be applicable even for the present system.

To examine the stoichiometry in the interaction between PLL and SCS, the $-[\theta]_{222}$ at low $\mathrm{pH}$ region, where both the $\varepsilon$-amino groups and the sulfonic groups are almost fully ionized, is plotted against the SCS mole fraction in Figure 5. For comparison, the stoichiometric relationship and the plot for the isotactic polymethacrylic acid

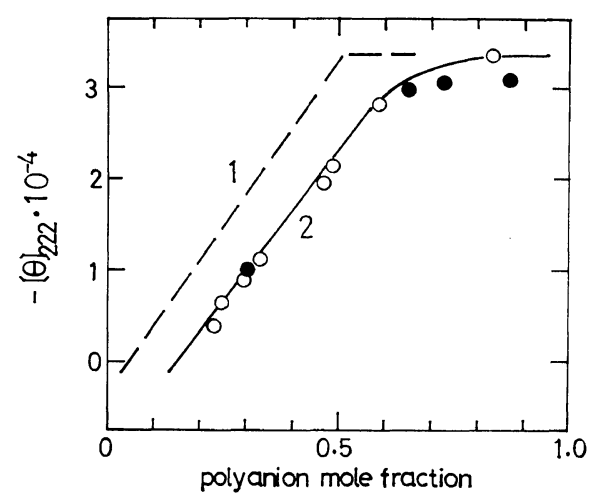

Figure 5. $-[\theta]_{222}$ plotted against polyanion mole fraction for PLL-SCS mixtures $(O)$ and for isotactic PMA-PLL mixtures (O). The Broken curve (1) denotes the stoichiometric relationship. 
(iso-PMA)-PLL system ${ }^{3}$ are also shown. The mole fraction of iso-PMA was corrected by the degree of dissociation $\alpha$ of the PMA. The plot for PLL-SCS is linear, but deviates from the stoichiometric relationship. A similar behavior was observed in the iso-PMA-PLL system (see filled circles in curve 2 in Figure 5). Poly(acrylic acid) stoichiometrically ${ }^{2}$ reacts with PLL, whence, the experimental points fall on curve 1 in Figure 5. One of the reasons for the difference in stoichiometry may be attributed to the difference in stiffness of the polymer chain in aqueous solution. In the PLL-SCS complex, the stiffness of the pyranose ring of SCS may lead to the formation of some free sulfonic groups which do not participate in the interaction with the $\varepsilon$ amino groups of PLL. No disturbing effect on the formation of $\alpha$-helix of PLL was observed in the region of large excess SCS mole fraction, in common with the iso-PMA-PLL system. This may also be explained by the location of the ionic groups.

Comparing these results with those reported by Gelman, et al., , ${ }^{4-8}$ for mucopolysaccharidesPLL systems, the following features can be pointed out: 1) the value of $-[\theta]_{222}$ for mucopolysaccharide-PLL system changes progressively with the mole ratio of component polymers and the maximum appeares at a characteristic mole ratio for each mucopolysaccharide, whereas, in the SCS-PLL system, $-[\theta]_{222}$ increases linearly with SCS mole fraction until all the PLL form $\alpha$-helices, and thereafter the value remains essentially constant. 2) Some of the CD spectra of the mucopolysaccharide-PLL systems show different patterns from that of PLL in aqueous solution, while all the CD spectra of the SCSPLL system are virtually the same as those of PLL. These differences in behavior between the SCS-PLL and the mucopolysaccharides-PLL systems seem to be analogous to those between PAA-PLL or isotactic PMA-PLL and atactic PMA-PLL systems.

We have pointed out the importance of the flexibility of the backbone chains and of the location of charged groups for the interaction of PLL with PAA and PMA. ${ }^{2,3}$ This consideration may be applicable to interpret the results for the polysaccharide-PLL systems.

SCS is a polymer of $\beta(1,4)$-D-glucopyranose

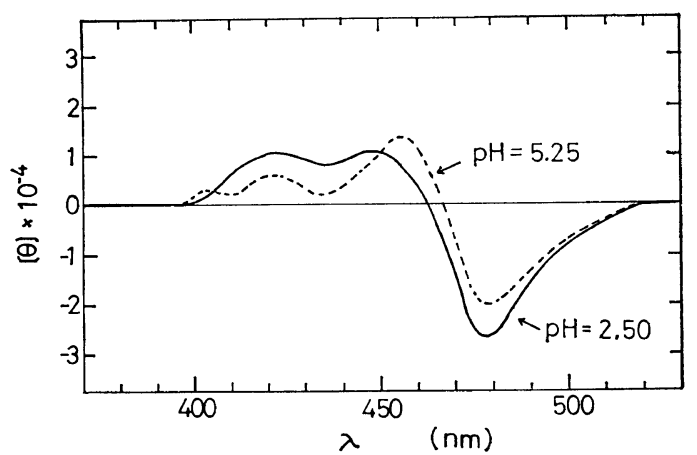

Figure 6. CD spectra of SCS-AO system. Molar ellipticity was calculated based on AO molar concentration: $[\mathrm{SCS}]=0.0038 \mathrm{~N} ;[\mathrm{AO}]=0.00013 \mathrm{~N}$.

sulfate. The conformation of SCS in aqueous solution is as yet poorly characterized. Figure 6 shows the induced CD spectra of the SCSacridine orange system in aqueous solution. The curves suggest that SCS can take at least a locally right-handed helical structure in aqueous solution. If we assume that SCS forms a fully extended structure, the average distance between the sulfonic groups on the SCS chain estimated from the results of crystalline cellulose ${ }^{9}$ is $2.0 \AA$. This value is close to $1.5 \AA$, the length per amino acid residue in the $\alpha$-helix. If we further assume that SCS surrounds the core consisting of $\alpha$-helix of PLL in a manner similar to some ordered super helical structure, we would be able to expect that the mean length per sulfonic group comes close to the value of $1.5 \AA$. This agreement of the mean distance between neighboring ionized groups would be one of the important supports for the conformation-directing interactions.

We conclude, through a series of studies on the conformation-directing interactions between poly-L-lysine and various polyacids, that the mutual location of oppositely charged groups closely relates to the chain conformations, and the configurations of the componont polyelectrolytes are decisive factors in discussing the interactions between these two polymer components.

\section{REFERENCES}

1. A. Nakajima, K. Shinoda, T. Hayashi, and H. Sato, Polymer J., 7, 550 (1975) 


\section{K. Shinoda, T. Hayashi, and A. Nakajima}

2. K. Shinoda, T. Hayashi, T. Yoshida, K. Sakai, and A. Nakajima, ibid., 8, 202 (1976).

3. K. Shinoda, K. Sakai, T. Hayashi, and A. Nakajima, ibid., 8, 208 (1976).

4. R. A. Gelman, W. B. Rippon, and J. Blackwell, Biopolymers, 12, 54 (1973).

5. R. A. Gelman, D. N. Glaser, and J. Blackwell, ibid., 12, 1223 (1973)
6. R. A. Gelman and J. Blackwell, ibid., 12, 1959 (1973).

7. R. A. Gelman and J. Blackwell, Arch. Biochem. Biophys., 159, 427 (1973).

8. R. A. Gelman and J. Blackwell, Biopolymers, 13, 139 (1974).

9. H. J. Wellard, J. Polym. Sci., 13, 471 (1954). 